\title{
Observation of Structural Anisotropy and the Onset of Liquidlike Motion During the Nonthermal Melting of InSb
}

\author{
K. J. Gaffney, ${ }^{1}$ A. M. Lindenberg, ${ }^{1}$ J. Larsson, ${ }^{2}$ K. Sokolowski-Tinten, ${ }^{3,4}$ C. Blome, ${ }^{5}$ O. Synnergren, ${ }^{2}$ J. Sheppard, ${ }^{6}$ \\ C. Caleman, ${ }^{7}$ A. G. MacPhee, ${ }^{8}$ D. Weinstein, ${ }^{8}$ D. P. Lowney, ${ }^{8}$ T. Allison, ${ }^{8}$ T. Matthews, ${ }^{8}$ R. W. Falcone,${ }^{8}$
}

A. L. Cavalieri, ${ }^{9}{ }^{10}$ D. M. Fritz, ${ }^{9}$ S. H. Lee, ${ }^{9}$ P. H. Bucksbaum, ${ }^{9}$ D. A. Reis,${ }^{9}$ J. Rudati, ${ }^{11}$ A. T. Macrander,${ }^{11}$ P. H. Fuoss, ${ }^{12}$ C. C. Kao, ${ }^{13}$ D. P. Siddons, ${ }^{13}$ R. Pahl,${ }^{14}$ K. Moffat,${ }^{14}$ J. Als-Nielsen, ${ }^{15}$ S. Duesterer, ${ }^{5}$ R. Ischebeck, ${ }^{5}$ H. Schlarb, ${ }^{5}$ H. Schulte-Schrepping, ${ }^{5}$ J. Schneider, ${ }^{5}$ D. von der Linde, ${ }^{4}$ O. Hignette, ${ }^{16}$ F. Sette, ${ }^{16}$ H. N. Chapman, ${ }^{17}$ R. W. Lee, ${ }^{17}$ T. N. Hansen, ${ }^{2}$ J. S. Wark, ${ }^{6}$ M. Bergh ${ }^{7}$ G. Huldt, ${ }^{7}$ D. van der Spoel,${ }^{7}$ N. Timneanu, ${ }^{7}$ J. Hajdu, ${ }^{7}$ R. A. Akre, ${ }^{18}$ E. Bong, ${ }^{18}$ P. Krejcik, ${ }^{18}$ J. Arthur, ${ }^{1}$ S. Brennan, ${ }^{1}$ K. Luening, ${ }^{1}$ and J. B. Hastings ${ }^{1}$

${ }^{1}$ Stanford Synchrotron Radiation Laboratory/SLAC, Menlo Park, California 94025, USA

${ }^{2}$ Department of Physics, Lund Institute of Technology, P.O. Box 118, S-22100, Lund, Sweden

${ }^{3}$ Institut für Optik und Quantenelektronik, Friedrich-Schiller Jena, Max-Wien-Platz 1, 07743 Jena, Germany

${ }^{4}$ Institut für Experimentelle Physik, Universität Duisburg-Essen, D-45117 Essen, Germany

${ }^{5}$ Deutsches Elektronen-Synchrotron DESY, Notkestrasse 85, 22607 Hamburg, Germany

${ }^{6}$ Department of Physics, Clarendon Laboratory, Parks Road, University of Oxford, Oxford OX1, 3PU, United Kingdom

${ }^{7}$ Department of Cell and Molecular Biology, Biomedical Centre, Uppsala University, SE-75124 Uppsala, Sweden

${ }^{8}$ Department of Physics, University of California, Berkeley, California 94720, USA

${ }^{9}$ FOCUS Center, Departments of Physics and Applied Physics Program, University of Michigan, Ann Arbor, Michigan 48109, USA

${ }^{10}$ Max-Planck-Institute of Quantum Optics, Hans-Kopfermann-Str. 1, D-85748 Garching, Germany

${ }^{11}$ Advanced Photon Source, Argonne National Laboratory, Argonne, Illinois 60439, USA

${ }^{12}$ Materials Science Division, Argonne National Laboratory, Argonne, Illinois 60439, USA

${ }^{13}$ National Synchrotron Light Source, Brookhaven National Laboratory, Upton, New York 11973, USA

${ }^{14}$ Consortium for Advanced Radiation Sources, The University of Chicago, Chicago, Illinois 60637, USA

${ }^{15}$ Niels Bohr Institute, Copenhagen University, 2100 Copenhagen Ø, Denmark

${ }^{16}$ European Synchrotron Radiation Facility, 38043 Grenoble Cedex 9, France

${ }^{17}$ Physics Department, Lawrence Livermore National Laboratory, Livermore, California 94550, USA

${ }^{18}$ Stanford Linear Accelerator Center, Menlo Park, California 94025, USA

(Received 7 June 2005; published 12 September 2005)

The melting dynamics of laser excited InSb have been studied with femtosecond x-ray diffraction. These measurements observe the delayed onset of diffusive atomic motion, signaling the appearance of liquidlike dynamics. They also demonstrate that the root-mean-squared displacement in the [111] direction increases faster than in the [110] direction after the first $500 \mathrm{fs}$. This structural anisotropy indicates that the initially generated fluid differs significantly from the equilibrium liquid.

DOI: 10.1103/PhysRevLett.95.125701

Intense femtosecond (fs) laser excitation of semiconductor crystals provides a pathway to crystal melting where the time dependent evolution of the atomic structure can be monitored directly with fs x-ray diffraction [1-7]. Improvements in ultrafast $x$-ray and electron sources have generated the most recent advances in our understanding of the dynamics of light driven phase transitions $[4,5,7,8]$. While pioneering ultrafast $\mathrm{x}$-ray diffraction experiments utilizing ultrafast laser plasma sources have shown that crystals disorder faster than the rate of electron-phonon coupling, the time needed to generate liquidlike structure and dynamics remains unclear [4,5]. Linear electron accelerators provide an alternative approach to ultrafast hard $\mathrm{x}$-ray generation that circumvents the fluence limitations of laser plasma sources and the long pulse durations of synchrotrons. This approach has been used for the SubPicosecond Pulse Source (SPPS) at the Stanford Linear Accelerator Center to generate fs hard x-ray pulses [9].
PACS numbers: 64.70.Dv, 61.10.-i, 63.20.Kr, 78.47.+p

Initial studies of ultrafast laser melting using the SPPS $\mathrm{x}$-ray source demonstrated that the initial stage of crystal disordering results from inertial motion on a laser softened potential energy surface [7]. The atoms initially sample the modified potential with velocities determined by the lattice temperature prior to laser excitation. These inertial dynamics dominate for the first half picosecond (ps) following laser excitation, indicating that interatomic forces minimally influence atomic excursions from the equilibrium lattice positions, even for motions in excess of an $\AA$. This also indicates that the atoms disorder initially without losing memory of their lattice reference.

In an attempt to identify the time needed to form a liquidlike structure and to better understand the mechanism for electronically driven melting in InSb, we extended our studies to longer delay times and a wider range of laser fluences. Following the Gaussian profile decay in the diffraction intensity, we observe an exponential decay. 
These biphasic dynamics occur for both Bragg peaks we measured and for laser fluences ranging from 50 to $130 \mathrm{~mJ} \mathrm{~cm}^{-2}$. Atomic diffusion represents the most reasonable explanation for this exponential decay and provides a clear experimental signature for liquidlike motion. After the first $500 \mathrm{fs}$, the root-mean-square displacement (rmsd) in the [111] direction increases with a rate larger than the rate in the [110] direction. The observation of anisotropy during the phase transition indicates that the initially formed liquid structure differs significantly from the equilibrium liquid and provides a direct test of the theoretical model potentials used to describe electronically driven melting [1].

The success of these experiments relied on three critical attributes of the experimental setup: the $80 \mathrm{fs}$ full width at half maximum (FWHM) x-ray pulse duration [9], a crossed beam x-ray-laser geometry that allows a series of relative time delays to be measured with a single pulse pair [10,11], and the low divergence of the $\mathrm{x}$-ray beam that allows for a grazing incidence $x$-ray geometry [7]. The SPPS produces fs pulses at $8.9 \mathrm{keV}$ with $2 \times 10^{6} \mathrm{x}$-ray photons/pulse in a $200 \times 400 \mu \mathrm{m}$ spot in a $1.5 \%$ bandwidth. The energy spread of the x-ray source allows the measurement to be sensitive to changes in lattice order, but insensitive to changes in the lattice constant. The pump laser is an amplified Ti:Sapphire laser running at $800 \mathrm{~nm}$ with a pulse duration of 50 fs FWHM. The convolution of the laser pulse and x-ray pulse durations gives an instrument resolution of $100 \mathrm{fs}$ FWHM. The laser runs at a repetition rate actively stabilized to the $\mathrm{x}$-ray pulses, resulting in a shortterm timing jitter of $\sim 300 \mathrm{fs}$ rms between the $\mathrm{x}$-ray and laser pulses [12].

In order to overcome this intrinsic jitter, the relevant time evolution must be measured with a single $\mathrm{x}$-ray-laser pulse pair. We achieved this objective with a noncollinear $\mathrm{x}$-ray and laser beam geometry $[10,11]$. The $25^{\circ}$ difference in the incidence angles for the $\mathrm{x}$-ray and laser pulses cause them to sweep across the surface of the crystal with different rates, imprinting a range of pump-probe delays on the surface of the crystal. Imaging the spatial profile of the diffracted $\mathrm{x}$-ray intensity with a CCD array detector provides the time history around $t=0$ in a single shot [7].

Matching the laser and $\mathrm{x}$-ray penetration represents an important requirement for successfully studying electronically driven melting. This goal has been achieved with a grazing x-ray incidence angle of $0.4^{\circ}$ with respect to the physical surface of the asymmetrically cut InSb crystal. $\mathrm{X}$-ray absorption-, not extinction-limited penetration insures that the probe depth does not increase when the crystal disorders. At $0.4^{\circ}$ grazing incidence, $95 \%$ of the x-ray diffraction will come from within $140 \mathrm{~nm}$ of the surface.

Before discussing the structural dynamics accessed with these measurements, the time scale of electronic equilibration [13,14], ambipolar diffusion [15,16], and phonon emission [17,18] must be addressed because these processes will influence the carrier density and nonthermal melting requires excitation of roughly $10 \%$ of the valence electrons $[1,4,19]$. As will be briefly discussed, theoretical and experimental characterization of these processes support the conclusion that the $\mathrm{x}$-ray pulse for time delays up to a few ps probe a fairly uniformly excited sample with an electronic temperature upwards of $10000 \mathrm{~K}$ and a lattice temperature less than the $798 \mathrm{~K}$ melting point of InSb.

Laser absorption determines the original carrier density profile. We have used the parameters of Rousse et al. [4] to determine the laser absorption and electron-hole pair density as a function of penetration depth. Large electron-hole densities should increase the ambipolar diffusion constant from the low carrier value of $\sim 30 \mathrm{~cm}^{2} \mathrm{~s}^{-1}[15,16,20]$. For diffusion constants ranging from 50 to $300 \mathrm{~cm}^{2} \mathrm{~s}^{-1}$, diffusion will smooth the carrier distribution faster than the inertial structural dynamics without significantly reducing the carrier density in the x-ray probed volume during the first few ps. Auger recombination and impact ionization will also change the excitation density. Theory and experiment indicate that the Auger recombination should take tens of fs $[13,14]$. The carrier density after electronic equilibration should still exceed the theoretical and measured nonthermal melting threshold $[1,4,19]$ because the energy absorbed in the first $150 \mathrm{~nm}$ of InSb when irradiated with a $130 \mathrm{~mJ} \mathrm{~cm}^{-2}$ laser pulse supports an average equilibrium electron-hole pair concentration of $\sim 1 \times$ $10^{22} \mathrm{~cm}^{-3}$. This has been calculated using a simple approximation to the InSb conduction band density of states [21]. Cooling of the electronic system will be comparatively slow because the optical phonon emission time for electrons occurs with an average rate of 1-3 ps for the energy range populated at low carrier density, and theory indicates that carrier screening at high densities will reduce the emission rate $[13,17,18]$.

We have used a time-dependent Debye-Waller-like model to describe the diffraction intensity, $I(t)$, and extract a time dependent mean-square displacement (MSD), $\left\langle\Delta r(t)^{2}\right\rangle$. In this model, $I(t)=I_{0} \exp \left(-\frac{Q^{2}\left\langle\Delta r(t)^{2}\right\rangle}{3}\right)$ and $\Delta r(t)=\left(3 \frac{\ln I_{0}-\ln I(t)}{Q^{2}}\right)^{1 / 2}$, where $I_{0}$ is the diffraction intensity of the ground state crystal, $Q$ corresponds to the reciprocal lattice vector, and $\Delta r(t)$ represents the rmsd. Assuming the MSD maintains a normal distribution during the temporal evolution, the logarithm of the signal provides a measure of the time evolution of the MSD during the phase transition.

Figure 1 shows the time dependent diffraction for the (111) and (220) reflections with $130 \mathrm{~mJ} \mathrm{~cm}^{-2}$ and $50 \mathrm{~mJ} \mathrm{~cm}^{-2}$ laser fluences. The signal has been fit to an error function with the instrument response resolution convolved with $S(t)=A_{g} \exp \left(-\frac{t^{2}}{\tau_{g}^{2}}\right)+A_{e} \exp \left(-\frac{t}{\tau_{e}}\right)$. For the (111) reflection, $\tau_{g}=0.34 \pm 0.08$ ps and $\tau_{e}=2.4 \pm$ $0.1 \mathrm{ps}$, while $\tau_{g}=0.25 \pm 0.08 \mathrm{ps}$ and $\tau_{e}=1.7 \pm 0.4 \mathrm{ps}$ for the (220) reflection. These values represent the average 


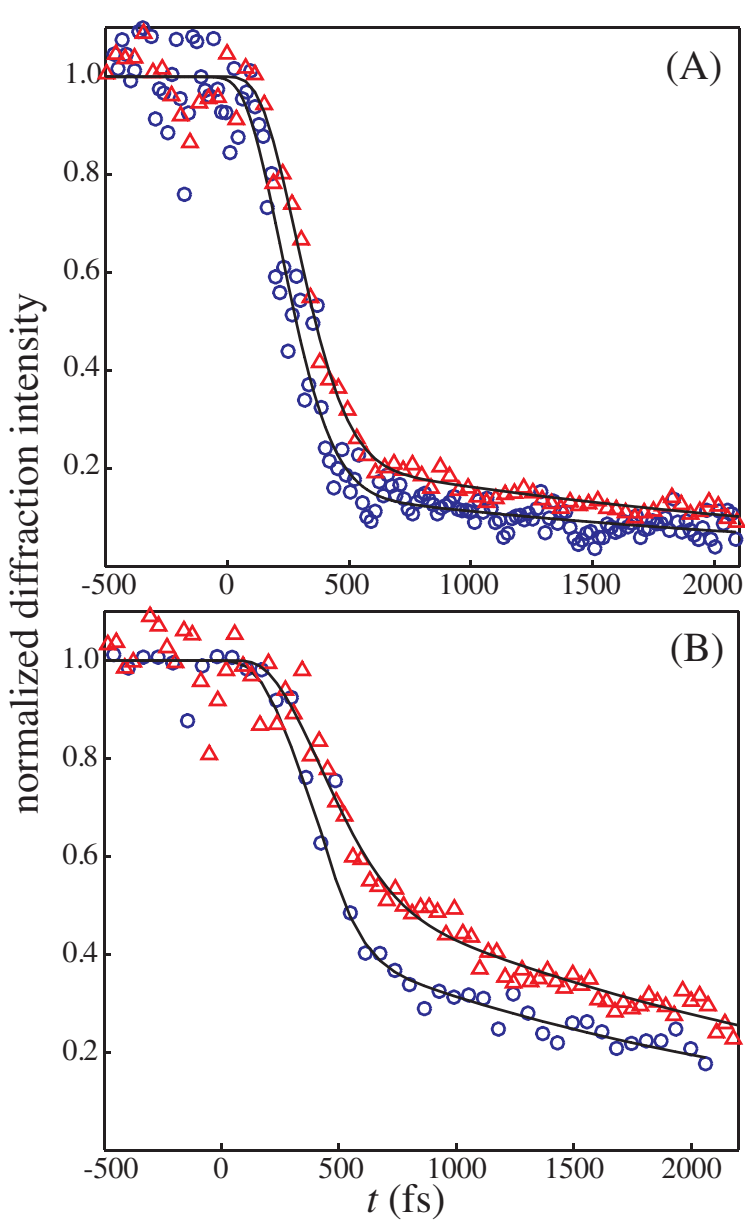

FIG. 1 (color online). Time dependent diffraction intensity for the $(111)(\triangle)$ and the $(220)(\bigcirc)$ reflections. Data collected with laser fluences of $130 \mathrm{~mJ} \mathrm{~cm}^{-2} 2$ (a) and $50 \mathrm{~mJ} \mathrm{~cm}^{-2}$ 2(b).

of the time constants extracted for laser fluences of 50, 90, and $130 \mathrm{~mJ} \mathrm{~cm}^{-2}$, since the amplitudes $A_{g}$ and $A_{e}$ represent the dominant source of laser fluence dependence in the signal. The time dependent rmsd appear in Fig. 2.

The time dependence of the MSD can be best understood by representing the MSD as a time integral over the velocity-velocity correlation function $\langle v(0) v(t)\rangle$ in two limiting conditions, a constant memory and a delta function memory [22]. Inertial dynamics correspond to constant memory, creating a rmsd that increases linearly with time, $\Delta r(t)=\left(\frac{3 k_{B} T}{M}\right)^{1 / 2} t$. This analysis predicts the Gaussian time constant to be $\tau_{g}=\left(\frac{M}{Q^{2} k_{B} T}\right)^{1 / 2}$, with values of $410 \mathrm{fs}$ and $250 \mathrm{fs}$ for the (111) and the (220) reflections, using a temperature of $300 \mathrm{~K}$ and a mass equal to the mean atomic mass for InSb [7]. These predictions lie within the 95\% confidence limits of the measured values. The amplitude of the inertial response indicates the magnitude of the lattice softening, since the lattice temperature remains constant during inertial motion and the MSD is inversely proportional to the average frequency of the thermally excited phonons. At the highest fluence studied, the inertial

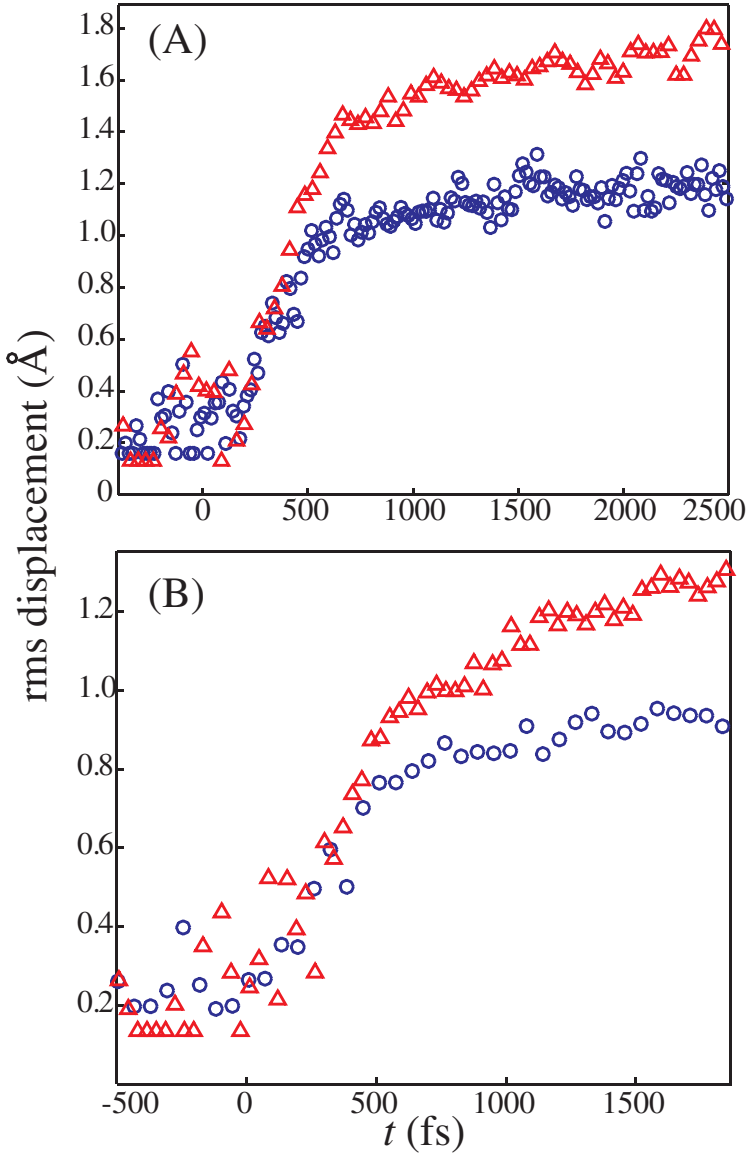

FIG. 2 (color online). Time dependent root-mean-square displacement for the (111) $(\triangle)$ and the $(220)(\bigcirc)$ reflections. Data collected with laser fluences of $130 \mathrm{~mJ} \mathrm{~cm}^{-2}$ 2(a) and $50 \mathrm{~mJ} \mathrm{~cm}^{-2}$ 2(b).

rmsd exceeds the ground state rmsd of $0.16 \AA$ by nearly an order of magnitude (Fig. 3).

Collisions will randomize the atomic velocities and cause $\langle v(0) v(t)\rangle$ to decay [22]. A delta function $\langle v(0) v(t)\rangle$ results in atomic diffusion, generates a diffraction intensity that decays exponentially, and signals the onset of liquidlike dynamics [23]. The exponential time constants used to fit the signal can be converted to a diffusion constant, $D=\frac{1}{2 Q^{2} \tau_{e}}$, with values of $7 \times 10^{-6} \mathrm{~cm}^{2} \mathrm{~s}^{-1}$ for the [111] direction and $4 \times$ $10^{-6} \mathrm{~cm}^{2} \mathrm{~s}^{-1}$ for the [110] direction. Using the EinsteinStokes equation and the temperature dependent viscosity of liquid InSb [24], we can estimate the temperature of the diffusing atoms to be in the range of 400 to $550 \mathrm{~K}$, supporting the conclusion that the thermal energy in the lattice remains significantly below the InSb melting point of $798 \mathrm{~K}$ during the electronically driven phase transition. While an intermediate time scale will exist where velocity correlations neither persist without change, nor decay instantaneously, the data does not support a more sophisticated analysis and the duration of this intermediate regime can be short lived in simple liquids $[22,25]$. 


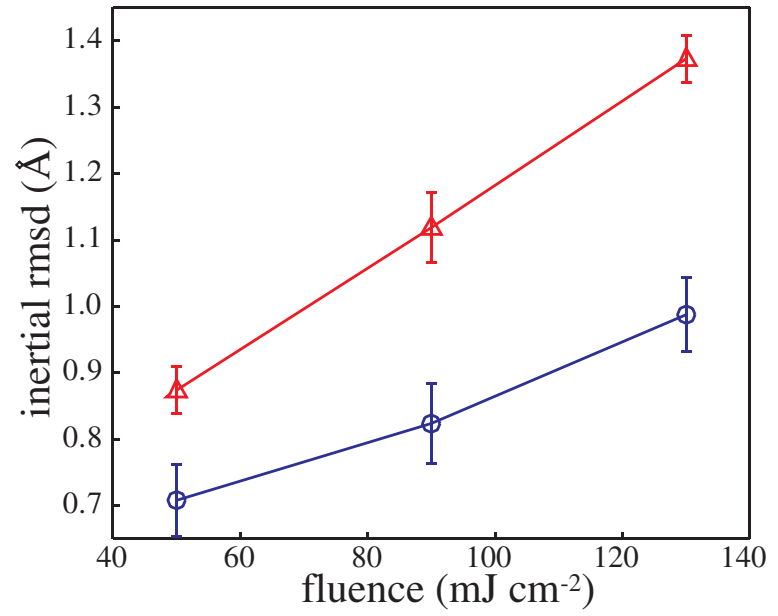

FIG. 3 (color online). Fluence dependence of the inertial rootmean-square displacement for the (111) $(\triangle)$ and the $(220)(\bigcirc)$ reflections.

The difference in the diffusion constant for the [111] and [110] directions indicates that the electron-hole plasma anisotropically modifies the potential, as does the relative magnitudes of the inertial rmsd in the [111] and the [110] directions (Fig. 3). Nor can these differences in diffusion times and inertial rmsd be explained with a hard sphere model of atomic collisions, because the [111] direction disorders faster despite the shorter interatomic distances.

Theoretical calculations indicate that the shear restoring force softens at the (111) zone boundary for Si at the lowest electron-hole plasma density $[1,19]$. Atomic disordering limited to planes transverse to the tetrahedral bonding directions would generate a larger rmsd in the [110] direction than in the [111], in direct contrast with the measurement. The observed anisotropy strongly suggests that the longitudinal optical phonons in the bonding directions, such as [111], also influence the phase transition pathway in nonthermal melting. Laser induced weakening of the bonding in InSb could also soften the longitudinal optical phonons in the directions parallel to the chemical bonds, creating anisotropy consistent with our experimental measurement. In the model of Stampfli and Bennemann, the longitudinal optical phonon in the [111] direction anharmonically couples to the transverse acoustic phonon, but does not directly soften [1]. This anharmonic coupling generates a significant delay in the longitudinal displacement of the atoms along the [111] direction. While this model appears unable to explain the anisotropy in the inertial response, discerning the relative contributions of lattice softening and atomic displacement would require measuring multiple Bragg peaks in the [111] direction in reciprocal space.

These studies of electronically driven melting in $\mathrm{InSb}$ demonstrate that an anisotropic fluid forms before the generation of the equilibrium liquid. The theory of phase nucleation developed by Oxtoby claims the change in symmetry and density that occurs during melting can lead to nucleating structures quite distinct from the equilibrated liquid [26,27]. While electronically driven melting appears to proceed along a barrierless pathway, the same discontinuities in the structure of the crystalline and liquid phases may require the structure of the initially formed fluid to differ from the equilibrium liquid in a fashion similar to that found in the critical nucleus.

Portions of this research were supported by the U.S. Department of Energy, Office of Basic Energy Science through direct support for the SPPS, as well as individual investigators and SSRL, a national user facility operated by Stanford University. Additional support for the construction of SPPS was provided in part by Uppsala University and the Swedish Research Council. Portions of this work were supported by a grant from the W. M. Keck Foundation. K. S. T., D. V. D. L., and S. T. gratefully acknowledge financial support by the Deutsche Forschungsgemeinschaft. J.L., O.S., T.N.H., K.S.T., and D. V.D.L. acknowledge the support of the European Commission through the FEMTO, $x$-RAY FEL PUMP-PROBE, and XPOSE projects. J.L. acknowledges support from Wallenberg Research Link and The Swedish Foundation for Strategic Research.

[1] P. Stampfli and K. H. Bennemann, Phys. Rev. B 49, 7299 (1994).

[2] K. Sokolowski-Tinten, J. Bialkowski, and D. von der Linde, Phys. Rev. B 51, 14186 (1995).

[3] I. L. Shumay and U. Hofer, Phys. Rev. B 53, 15878 (1996).

[4] A. Rousse et al., Nature (London) 410, 65 (2001).

[5] K. Sokolowski-Tinten et al., Phys. Rev. Lett. 87, 225701 (2001).

[6] S. K. Sundaram and E. Mazur, Nat. Mater. 1, 217 (2002).

[7] A. M. Lindenberg et al., Science 308, 392 (2005).

[8] B. J. Siwick, J. R. Dwyer, R. E. Jordan, and R. J. D. Miller, Science 302, 1382 (2003).

[9] P. Krejcik et al., in Proceeding of the 2003 Particle Accelerator Conference, edited by J. Chew, S. Lucas, and S. Webber (IEEE, Piscataway, NJ, IEEE, Portland, OR, 2003), p. 423.

[10] R. Neutze and J. Hajdu, Proc. Natl. Acad. Sci. U.S.A. 94, 5651 (1997).

[11] O. Synnergren et al., Appl. Phys. Lett. 80, 3727 (2002).

[12] A. L. Cavalieri et al., Phys. Rev. Lett. 94, 114801 (2005).

[13] E. J. Yoffa, Phys. Rev. B 21, 2415 (1980).

[14] P. M. Fauchet, Phys. Status Solidi B 110, K11 (1982).

[15] J.F. Young and H. M. van Driel, Phys. Rev. B 26, 2147 (1982).

[16] H. Bergner, V. Bruckner, and M. Schubert, J. Lumin. 30, 114 (1985).

[17] T. Grave, E. Scholl, and H. Wurz, J. Phys. C 16, 1693 (1983).

[18] E. J. Yoffa, Phys. Rev. B 23, 1909 (1981). 
[19] R. Biswas and V. Ambegaokar, Phys. Rev. B 26, 1980 (1982).

[20] K. Jarasiunas, S. Stonys, and E. Sirmulis, IEEE J. Quantum Electron. 22, 1341 (1986).

[21] J. R. Chelikowsky and M. L. Cohen, Phys. Rev. B 14, 556 (1976).

[22] G. D. Harp and B. J. Berne, Phys. Rev. A 2, 975 (1970).
[23] D. A. McQuarrie, Statistical Mechanics (HarperCollins, New York, 1976).

[24] Y. Sato et al., Int. J. Thermophys. 23, 235 (2002).

[25] R. M. Stratt, Acc. Chem. Res. 28, 201 (1995).

[26] D. W. Oxtoby, Acc. Chem. Res. 31, 91 (1998).

[27] Y.C. Shen and D. W. Oxtoby, J. Chem. Phys. 105, 6517 (1996). 